2019-07

Objectively measured sleep duration

and plasma glucose values following an

oral glucose tolerance test amongst

pregnant women with obesity in the UK.

Redfern, Kathy

http://hdl.handle.net/10026.1/13246

10.1016/j.sleep.2019.01.017

Sleep Medicine

Elsevier

All content in PEARL is protected by copyright law. Author manuscripts are made available in accordance with publisher policies. Please cite only the published version using the details provided on the item record or document. In the absence of an open licence (e.g. Creative Commons), permissions for further reuse of content should be sought from the publisher or author. 
Title: Objectively measured sleep duration and plasma glucose values following an oral glucose tolerance test amongst pregnant women with obesity in the UK.

Authors: Kathy M. Redfern ${ }^{1}$, Rachel S. Hine ${ }^{1}$, Heidi J. Hollands ${ }^{2}$, C. Ross Welch²; Jonathan H. Pinkney ${ }^{3}$, Gail A. Rees ${ }^{1}$

${ }^{1}$ School of Biomedical Sciences, Faculty of Medicine and Dentistry, University of Plymouth, United Kingdom. ${ }^{2}$ Maternity Department, University Hospitals Plymouth NHS Trust, United Kingdom, ${ }^{3}$ Institute of Translational \& Stratified Medicine, Faculty of Medicine and Dentistry, University of Plymouth, United Kingdom.

Corresponding author: Dr Kathy Redfern. Address: School of Biomedical Sciences, Plymouth University, Drake Circus, Plymouth PL4 8AA. Email: kathy.redfern@ plymouth.ac.uk. Telephone: +44 1752586901

Conflict of Interest: The authors declare no conflict of interest. 


\section{Objectively measured sleep duration and plasma glucose values following an oral glucose tolerance test amongst pregnant women with obesity in the UK.}

\section{Abstract}

Background/objectives: Short sleep duration has been linked to maternal hyperglycaemia following a 1-hour 50g oral glucose tolerance test (OGTT) in observational studies conducted primarily in the USA. Our objective was to examine the relationship between objectively measured sleep duration and plasma glucose values following the 2-hour 75g OGTT routinely used in the UK amongst women with obesity.

Methods: Sleep and OGTT data were available for 49 pregnant women who wore wrist actigraphs for at least 2 nights, and took a 2-hour 75g OGTT at the end of their second trimester. Multivariable regression was used to evaluate associations between sleep duration and OGTT results.

Results: Higher 2-hour plasma glucose values were significantly associated with shorter sleep duration and higher maternal age and BMI, with no association observed between wake after sleep onset (WASO) and 2-hour plasma glucose values. No associations were observed between fasting plasma glucose values and any sleep parameters.

Conclusions: We found that shorter sleep duration, as measured using actigraphy, is associated with higher 2-hour plasma glucose values following a 2-hour 75g OGTT in the UK. These findings are in keeping with those observed elsewhere in the world, employing alternative OGTT protocols. Future studies should investigate sleep extension as a potential candidate for inclusion in future RCTs aiming to improve glucose tolerance and prevent GDM amongst those at risk.

Keywords: Pregnancy, sleep duration, glucose tolerance, obesity, actigraphy.

\section{Introduction}

It is well documented that maternal obesity, excessive gestational weight gain and the development of gestational diabetes mellitus are risk factors for multiple adverse maternal and infant outcomes during pregnancy [1-3]. Numerous maternal lifestyle interventions aiming to limit gestational weight gain, prevent gestational diabetes mellitus and improve other maternal and infant outcomes have been conducted to date, with the majority of studies focusing on diet and physical activity $[4,5]$. Sleep is another potentially modifiable maternal lifestyle factor, and short sleep duration has been associated with metabolic disturbances and increased risk of obesity and type 2 diabetes in nonpregnant populations [6]. A recent meta-analysis of individual patient data demonstrated that objectively measured short sleep duration during pregnancy ( $<6.5$ hours) was associated with higher 
1-hour glucose levels following a 50g oral glucose tolerance test (OGTT) and increased risk of gestational diabetes melitus [7]. However, of the four studies included in analysis, three measured sleep duration using a single overnight polysomnography (PSG), which as the authors acknowledge, may not reflect habitual sleep duration as measured by actigraphy, which was reported for just one study.

The diagnostic criteria for gestational diabetes mellitus varies worldwide, as does the dose of glucose administered as part of an OGTT. In the UK, the National Institute for Health and Care Excellence (NICE) recommend the use of the 2-hour 75g OGTT to test for gestational diabetes mellitus in women with known risk factors [8]. The studies included in Reutrakul and colleagues' meta-analysis [7] were conducted in the US or Canada with different diagnostic criteria for gestational diabetes mellitus. To our knowledge, no studies have examined the impact of objectively measured sleep duration on blood glucose values following a $75 \mathrm{~g} 2$-hour OGTT according to NICE guidelines in the UK. It is important to ascertain whether a relationship exists before sleep is considered for incorporation into RCTs or antenatal advice in the UK. The aim of the present study is to investigate the impact of sleep duration assessed using actigraphy on OGTT results amongst a cohort of women with obesity in Plymouth, UK.

\section{Materials and methods}

\subsection{Study population and design}

Participants in the present study were part of a larger observational study examining the impact of GWG, diet and physical activity on maternal and infant outcomes, the results of which are described elsewhere (in press).

Inclusion criteria included: age $\geq 18$ and $<40$ years, $\mathrm{BMI} \geq 30$ and $<40 \mathrm{~kg} / \mathrm{m}^{2}$, gestation $\leq 12$ weeks at first antenatal appointment and a singleton pregnancy. Women with multiple pregnancies, preexisting type I or type II diabetes mellitus or women identifying as any ethnicity other than white Caucasian were not eligible to participate, as the study was not powered to detect differences between these groups. Eligible women were identified from their first antenatal appointment notes and approached at their 12 week dating scan at Derriford Hospital in Plymouth, UK. Ethical approval was obtained from the NHS Health Research Authority National Research Ethics Service and local approval was obtained from Plymouth Hospitals NHS Trust.

\subsection{Data collection}

Following recruitment, verbal and written informed consent was obtained, and a questionnaire was administered to participants between 12 and 14 weeks gestation. 
In the present study, sleep data were collected at the end of the second trimester. Women wore an Actigraph wrist-worn accelerometer (Actigraph wGT3X-BT, Florida, USA) on their dominant wrist for four days following the study visit, after which the device was analysed on the corresponding computer software program (Actilife 6.0, Florida, USA).

Participants with $<2$ valid days of sleep data were excluded from analyses. Sleep was identified using the Cole-Kripke sleep scoring algorithm [9] which has been validated for use in an adult population and recently used to assess sleep duration and disruption in late pregnancy [10]. For each participant, mean sleep duration, mean wake after sleep onset (WASO), WASO as a percentage of total sleep and sleep fragmentation was reported. WASO is defined as the total minutes spent awake between the onset of sleep and the end of rest interval, while sleep fragmentation is an index of restlessness during the sleep period expressed as a percentage.

All women enrolled in the study received a routine 2 -hour $75 \mathrm{~g}$ OGTT at approximately 28 weeks, as per NICE guidance [8]. Fasting and 2-hour plasma glucose values were reported in mmol and the incidence of gestational diabetes mellitus according to NICE diagnostic criteria was recorded for each participant.

\subsection{Statistical analysis}

All data were entered into and analysed using SPSS (Statistics Package for the Social Sciences) for Windows version 21 (IBM, Chicago USA). The level of significance was set to a probability $(p)<0.05$ for all statistical tests performed. Multiple regression was used to evaluate the extent to which maternal sleep duration and WASO influence maternal fasting and 2-hour plasma glucose values following an OGTT after adjustment for age and BMI at first antenatal appointment. Sleep fragmentation was not included in the model in order to avoid multi-collinearity, as it was correlated with WASO.

\section{Results}

Of 75 women enrolled in the study, 49 had at least 2 days of valid sleep data in trimester two and an OGTT result. Table 1 shows the descriptive characteristics of these women. Women slept for an average of $7.0 \pm 0.3$ hours per night and the average time spent in WASO was $9.0 \pm 4.3 \%$. Ten of the 49 women were diagnosed with GDM according to UK NICE guidance. 


\begin{tabular}{|c|c|}
\hline & $n=49$ \\
\hline Age, years & $30.0 \pm 4.4$ \\
\hline BMI, kg/m ${ }^{2}$ & $33.2 \pm 2.1$ \\
\hline Nulliparous, n (\%) & $20(40.8)$ \\
\hline $\begin{array}{l}\text { Median gestation at sleep } \\
\text { data collection, weeks }{ }^{+ \text {days }} \\
\text { (IQR) }\end{array}$ & $28^{+4}\left({ }^{5.5}\right)$ \\
\hline Fasting glucose, mmol/l & $4.6 \pm 0.6$ \\
\hline 2-hour glucose, mmol/l & $6.3 \pm 1.6$ \\
\hline GDM diagnosis, n (\%) & $10(20.4)$ \\
\hline Sleep duration, hours & $7.0 \pm 0.3$ \\
\hline WASO, minutes & $35.2 \pm 17.1$ \\
\hline WASO, $\%$ & $9.0 \pm 4.3$ \\
\hline Sleep fragmentation index, \% & $27.4 \pm 14.9$ \\
\hline \multicolumn{2}{|c|}{$\begin{array}{l}\text { Mean } \pm \text { SD unless otherwise stated } \\
\text { IQR: interquartile range. } \\
\text { GDM: gestational diabetes mellitus }\end{array}$} \\
\hline
\end{tabular}

No statistically significant associations were found between maternal age, BMI, sleep duration or WASO and fasting plasma glucose values. Higher 2-hour plasma glucose values were significantly associated with shorter sleep duration, higher maternal age and higher maternal BMI, with no association observed between WASO and 2-hour plasma glucose values (Table 2).

Table 2 Summary of multiple regression analyses for associations between blood glucose, maternal age, BMI, sleep duration and WASO.

\begin{tabular}{llll|lll}
\hline \multicolumn{5}{c|}{ Fasting glucose } & & \multicolumn{4}{l}{ 2-hour glucose } & \\
\hline & $\beta$ & $95 \% \mathrm{Cl}$ & $\mathrm{p}$ & $\beta$ & $95 \% \mathrm{Cl}$ & $\mathrm{p}$ \\
\hline $\begin{array}{l}\text { Maternal age, } \\
\text { years }\end{array}$ & 0.11 & $-0.027-0.058$ & 0.477 & 0.41 & $0.045-0.246$ & 0.006 \\
$\mathrm{BMI}, \mathrm{kg} / \mathrm{m}^{2}$ & 0.21 & $-0.026-0.145$ & 0.165 & 0.28 & $-0.011-0.414$ & 0.036 \\
\hline $\begin{array}{l}\text { Sleep duration, } \\
\text { minutes }\end{array}$ & -0.05 & $-0.002-0.002$ & 0.777 & -0.35 & $-0.009--0.001$ & 0.025 \\
\hline WASO, \% & 0.05 & $-0.043-0.057$ & 0.774 & -0.04 & $-0.131-0.103$ & 0.809 \\
\hline
\end{tabular}

\section{Discussion}

Results from our study indicate that sleep duration is inversely associated with 2-hour, but not fasting plasma glucose values, following a 2-hour 75g OGTT, even after adjustment for maternal age, $\mathrm{BMI}$ and WASO. To our knowledge, this is the first study to examine the impact of objectively 
measured sleep duration using actigraphy on plasma glucose values amongst women in the UK, who receive a 2-hour 75g OGTT according to NICE guidelines [8].

Findings from our study are similar to those of Herring et al. [11] who observed that objectively measured short sleep duration using actigraphy was associated with incidence of gestational diabetes mellitus and higher 1-hour plasma glucose values following a 50g-OGTT in their cohort of 63 low-income urban women in the US. Other studies using self-reported measures of sleep duration have also reported an inverse relationship [12], and in some cases a U-shaped relationship between sleep duration and incidence of gestational diabetes mellitus [13]. Findings from the present study therefore suggest that the relationship between sleep duration and glucose intolerance is similar for women in the UK undergoing a 75g 2-hour OGTT to those in the US, who have typically undergone a 50g 2-hour OGTT in the current literature [7].

Experimental data from non-pregnant populations has demonstrated adverse effects on glucose tolerance, insulin response, insulin sensitivity and glucose disposal with short sleep, but not regular sleep $[14,15]$. To our knowledge, no such experimental data has been collected from women during pregnancy, but Twedt and colleagues are currently conducting a pilot RCT to determine whether sleep education during pregnancy improves glycemic control amongst women with gestational diabetes mellitus in Pittsburgh, US [16].

A strength of the present study is that sleep duration has been measured objectively using actigraphy. Actigraphy correlates well with PSG and enables the assessment of habitual sleep in research participants' homes rather than in the laboratory [17]. Actigraphy has also been shown to be preferable to self-report questionnaires for estimation of sleep duration during pregnancy [18]. The accelerometers were programmed to collect data from 00.00 to 00.00 over four days, as a primary aim of the study was to collect 4 days of physical activity data. This meant that two nights of potential sleep data collection were interrupted, so that only a maximum of three night's data were available for each participant. 
The present study was conducted amongst a cohort of women with obesity, with a BMI $\geq 30$ and < $40 \mathrm{~kg} / \mathrm{m}^{2}$. Obesity is a well-documented risk factor for impaired glucose metabolism during pregnancy and GDM [2], and the majority of intervention studies aiming to prevent gestational diabetes mellitusvhave been conducted amongst this group of women [19]. The women in this cohort were all receiving antenatal care in Plymouth, UK, where the majority of women are White Caucasian. As such, only women from this group were eligible to participate, as the study was not powered to identify differences between other ethnic groups without recruiting a larger sample of women, which was not feasible. As the prevalence of gestational diabetes mellitus is well-known to vary between different ethnic groups [20], the findings from the present study cannot be generalised beyond the present population. Future work in a larger cohort should include women of different ethnicities and from different geographical areas of the UK. It was also not possible to include daytime sleeping in our analysis as the majority of women did not sleep during the day, and thus the study was not powered to examine whether this practice influenced plasma glucose values. This is something that should be examined in future work, in a larger cohort.

In summary, we found that shorter sleep duration, as measured using actigraphy, is associated with higher 2-hour plasma glucose values following a 2-hour 75g OGTT in the UK, after adjustment for age, maternal BMI and WASO. These findings are in keeping with those observed elsewhere in the world, with different OGTT protocols, and thus suggest sleep extension as a potential candidate for inclusion in future RCTs aiming to improve glucose tolerance and prevent gestational diabetes mellitus amongst those at risk. However, since long sleep duration has also been associated with impaired glucose tolerance and risk of gestational diabetes mellitus, larger, more diverse studies using objective measures of sleep duration are first required in order to identify optimal sleep duration during pregnancy. 


\section{Acknowledgments.}

R.S received a bursary from the Nutrition Society in the UK in order to undertake a summer placement within the research group. The rest of the research team did not receive any funding to undertake this research.

\section{References}

[1] O'Sullivan EP, Avalos G, O'Reilly M, Dennedy MC, Gaffney G, Dunne F. Atlantic Diabetes in Pregnancy (DIP): the prevalence and outcomes of gestational diabetes mellitus using new diagnostic criteria. Diabetologia 2011;54:1670-5. doi:10.1007/s00125-011-2150-4.

[2] Scott-Pillai R, Spence D, Cardwell CR, Hunter A, Holmes VA. The impact of body mass index on maternal and neonatal outcomes: a retrospective study in a UK obstetric population, 20042011. Br J Obstet Gynaecol 2013;120:932-9. doi:10.1111/1471-0528.12193.

[3] Mamun A, Callaway LK, O'Callaghan MJ, Williams GM, Najman JM, Alati R, et al. Associations of maternal pre-pregnancy obesity and excess pregnancy weight gains with adverse pregnancy outcomes and length of hospital stay. BMC Pregnancy Childbirth 2011;11:62. doi:info:pmid/21892967.

[4] Muktabhant B, Lawrie TA, Lumbiganon P, Laopaiboon M. Diet or exercise, or both, for preventing excessive weight gain in pregnancy. Cochrane Database Syst Rev 2015:CD007145. doi:10.1002/14651858.CD007145.pub3.

[5] Shepherd E, Gomersall JC, Tieu J, Han S, Crowther CA, Middleton P. Combined diet and exercise interventions for preventing gestational diabetes mellitus. Cochrane Database Syst Rev 2017;11:CD010443. doi:10.1002/14651858.CD010443.pub3.

[6] Knutson KL, Van Cauter E. Associations between sleep loss and increased risk of obesity and diabetes. Ann N Y Acad Sci 2008;1129:287-304. doi:10.1196/annals.1417.033.

[7] Reutrakul S, Anothaisintawee T, Herring SJ, Balserak BI, Marc I, Thakkinstian A. Short sleep duration and hyperglycemia in pregnancy: Aggregate and individual patient data metaanalysis. Sleep Med Rev 2018;40:31-42. doi:10.1016/J.SMRV.2017.09.003.

[8] National Institute for Health and Care Excellence. Diabetes in pregnancy: managementfrom preconception to the postnatalperiod. 2015.

[9] Cole RJ, Kripke DF, Gruen W, Mullaney DJ, Gillin JC. Automatic sleep/wake identification from wrist activity. Sleep 1992;15:461-9.

[10] Lee KA, Gay CL, Alsten CR. Sleep Enhancement Training for Pregnant Women. Obstet Gynecol 2016;128:964-71. doi:10.1097/AOG.0000000000001654.

[11] Herring SJ, Nelson DB, Pien GW, Homko C, Goetzl LM, Davey A, et al. Objectively measured sleep duration and hyperglycemia in pregnancy. Sleep Med 2014;15:51-5. doi:10.1016/j.sleep.2013.07.018.

[12] Qiu C, Enquobahrie D, Frederick IO, Abetew D, Williams MA. Glucose intolerance and gestational diabetes risk in relation to sleep duration and snoring during pregnancy: a pilot study. 2010.

[13] Rawal S, Hinkle SN, Zhu Y, Albert PS, Zhang C. A longitudinal study of sleep duration in pregnancy and subsequent risk of gestational diabetes: findings from a prospective, 
multiracial cohort 2017. doi:10.1016/j.ajog.2016.11.1051.

[14] Donga E, van Dijk M, van Dijk JG, Biermasz NR, Lammers G-J, van Kralingen KW, et al. A Single Night of Partial Sleep Deprivation Induces Insulin Resistance in Multiple Metabolic Pathways in Healthy Subjects. J Clin Endocrinol Metab 2010;95:2963-8. doi:10.1210/jc.2009-2430.

[15] Buxton OM, Pavlova M, Reid EW, Wang W, Simonson DC, Adler GK. Sleep Restriction for 1 Week Reduces Insulin Sensitivity in Healthy Men. Diabetes 2010;59:2126 LP-2133.

[16] ClinicalTrials.gov. Identifier: NCT03266133. Sleep Intervention to Improve Glycemic Control in Women With Gestational Diabetes. Natl Libr Med 2017. https://clinicaltrials.gov/ct2/show/NCT03266133.

[17] Marino M, Li Y, Rueschman MN, Winkelman JW, Ellenbogen JM, Solet JM, et al. Measuring sleep: accuracy, sensitivity, and specificity of wrist actigraphy compared to polysomnography. Sleep 2013;36:1747-55. doi:10.5665/sleep.3142.

[18] Herring SJ, Foster GD, Pien GW, Massa K, Nelson DB, Gehrman PR, et al. Do pregnant women accurately report sleep time? A comparison between self-reported and objective measures of sleep duration in pregnancy among a sample of urban mothers. Sleep Breath 2013;17:13237. doi:10.1007/s11325-013-0835-2.

[19] Song C, Li J, Leng J, Ma RC, Yang X. Lifestyle intervention can reduce the risk of gestational diabetes: a meta-analysis of randomized controlled trials. Obes Rev 2016;17:960-9. doi:10.1111/obr.12442.

[20] Yuen L, Wong VW. Gestational diabetes mellitus: Challenges for different ethnic groups. World J Diabetes 2015;6:1024-32. doi:10.4239/wjd.v6.i8.1024. 